\title{
Zodiacal light, certitudes and questions
}

\author{
A. Ch. Levasseur-Regourd \\ Univ. Paris VI/Aéronomie, B.P. 3, 91371 Verrières, France \\ (Received November 10, 1997; Revised December 12, 1997; Accepted January 9, 1998)
}

\begin{abstract}
Progress achieved in zodiacal light studies not only allows the estimation of the brightness and polarization to be expected in a given direction for an observer in the ecliptic plane; it also provides information about the radial dependence of some local optical properties, and about their phase curves. It is nevertheless necessary to stress the high level of the uncertainties, with, as an example, a value of $(0.15 \pm 0.08)$ derived from two approaches for the local geometric albedo at the Earth. Besides, various open questions remain, possibly because of the lack of space observations since the early eighties. This paper would like to warn the reader against crude extrapolations of the available results; it also emphasizes the need for further observations and new computational tools, and presents new laboratory measurements, such as the microgravity series of experiments now under development.
\end{abstract}

\section{Introduction}

The faint glow of the zodiacal light is produced by a sunlit lenticular cloud of dust, the density of which increases towards the Sun and near the ecliptic plane. During the second part of the twentieth century, enormous progress has been achieved in the studies of the zodiacal light, due to quantitative measurements and spaceborne observations, and to comparison with in-situ studies of interplanetary dust particles. However, it is still difficult to obtain a zodiacal light model which allows the observer to estimate accurately the "foreground noise" for space photometry of faint extended astronomical sources; such a model would furthermore not represent the evolution of the zodiacal light over millions or billions of years, and thus be useless for studies of the evolution of the solar system. These are precisely the reasons why we would like to present what is certain about the zodiacal light, to raise some questions, and to stress the topics that deserve further detailed studies, not only in terms of observations and light scattering computations, but also in terms of laboratory measurements.

What we call here zodiacal light is the sunlight scattered by interplanetary dust particles, whose color is nearly solar in the 0.2 to $3 \mu \mathrm{m}$ domain, in contrast with the thermal emission from these particles, which prevails in the infrared domain. For a given location of the observer and direction of the line-of-sight, the scattered light is described by its brightness and its polarization. Taking into account the overall shape of the zodiacal cloud, the direction of observation is usually defined by its helio-ecliptic coordinates.

\section{Zodiacal Light, What is Certain}

\subsection{Short period variations and symmetry plane at the} Earth

For an observer moving with the Earth, temporal variations in the zodiacal light brightness at a constant helio-

Copy right (C) The Society of Geomagnetism and Earth, Planetary and Space Sciences (SGEPSS); The Seismological Society of Japan; The Volcanological Society of Japan; The Geodetic Society of Japan; The Japanese Society for Planetary Sciences. ecliptic direction can be noticed. Yearly variations, quite conspicuous for high or medium ecliptic latitudes, certainly exist. They originate both in the slight inclination of the plane of symmetry of the cloud with respect to the ecliptic plane, and in the eccentricity of the Earth's orbit (Dumont and Levasseur-Regourd, 1978; Leinert et al., 1980). At the Earth's orbit, the symmetry plane is quite close to the invariant plane of the solar system, with an inclination of about $(1.5 \pm 0.4)^{\circ}$ and an ascending node near $(95 \pm 20)^{\circ}$. When proper corrections are made, the zodiacal light, as observed from a given observatory in a given direction, is approximately stable; no significant correlation is found with solar activity (Dumont and Levasseur-Regourd, 1978; Leinert and Pitz, 1989).

\subsection{Brightness and polarization in the ecliptic plane}

Once the effect of the position of the observer above or below the symmetry plane has been taken into account, the zodiacal light, as observed from the Earth, is described by tables which provide its brightness (either in magnitude related units or in SI units) and its degree of polarization (i.e. the partial linear polarization, by definition positive when the electric field vector is perpendicular to the scattering plane, and negative when it is parallel to it). From the extensive Dumont and Sanchez surveys (1975, 1976), in good agreement with space observations, a $5^{\circ}$ steps table has been proposed by Levasseur-Regourd and Dumont (1980). It has recently been extended to a larger sky coverage and to polarization data (Levasseur-Regourd, 1996; Leinert et al., 1998). For observations in the half space where the zodiacal foreground is low, that is to say for solar elongations greater than $90^{\circ}$, the brightness at $0.55 \mu \mathrm{m}$ remains between 8 and $26 \cdot 10^{-7} \mathrm{~W} \mathrm{~m}^{-2} \mathrm{sr}^{-1} \mu \mathrm{m}^{-1}$, and the degree of polarization between -3 and +21 percent; the uncertainties are smaller than $10^{-7} \mathrm{~W} \mathrm{~m}^{-2} \mathrm{sr}^{-1} \mu \mathrm{m}^{-1}$ for the brightness, and 2 percent for the polarization. A detailed discussion of the coordinate transformations, units, tables and uncertainties can be found in Leinert et al. (1998). It should be added that, away from the Earth in the ecliptic plane, the brightness decreases and the 
polarization increases with increasing solar distance R. In the inner solar system, the brightness variation can be described by a $\mathrm{R}^{-2.3}$ power law, and the polarization variation by a $\mathrm{R}^{+0.3}$ law (Leinert et al., 1982); in the outer solar system and at least up to the outer part of the asteroid belt, it decreases approximately as $\mathrm{R}^{-2.6}$ (Toller and Weinberg, 1985), or, with a bimodal population, as $R^{-2.5}$ up to $2.3 \mathrm{AU}$, and $\mathrm{R}^{-2.37}$ in the asteroid belt (Hanner et al., 1976).

\subsection{Radial dependence of local optical properties and density}

The above mentioned results, although essential to estimate the zodiacal foreground, do not provide information about the local properties of the dust: the measured values are integrated along a line-of-sight, which extends from the observer to the outer fringe of the zodiacal cloud. We have retrieved some local properties in the symmetry plane and at a constant phase angle of $90^{\circ}$, through the nodes of lesser uncertainty method (Dumont and Levasseur-Regourd, 1988; Levasseur-Regourd et al., 1991). As expected from the above mentioned variation in integrated polarization, the optical properties of the dust are far from being the same everywhere. Between 0.3 and 1.5 AU, the local polarization is found to vary as $\mathrm{R}^{+0.5 \pm 0.1}$, and the local albedo as $\mathrm{R}^{-0.34 \pm 0.05}$. Between 0.1 and $0.3 \mathrm{AU}$, a steeper slope is noticed for the local polarization; this trend, together with the extrapolated variation of the albedo derived from IRAS spacecraft data, agrees with the trends obtained by Mann $(1992,1996)$ in the F-Corona. For the overall evolution of the local density, a $\mathrm{R}^{-0.93 \pm 0.067}$ power law is retrieved, in agreement with the 1/R law, expected for dust under Poynting-Robertson drag. However, the circumsolar dust ring in resonant lock with the Earth (Dermott et al., 1994) induces a slight deviation from the density power law in the vicinity of the Earth's orbit (Renard et al., 1996).

\subsection{Local albedo and polarization}

The local albedo value retrieved at 1.5 AU, by a comparison of the brightness and IRAS thermal emission data, is in the 0.07 to 0.08 range. This value is at $90^{\circ}$ phase angle, while the geometric albedo is by definition given at $0^{\circ}$ phase angle. Taking into account the albedo phase curve at 1.5 AU (see figure 3-c in Renard et al., 1995), it leads to a value in the 0.17 to 0.20 range, for the geometric albedo at $1.5 \mathrm{AU}$. Large error bars can be suspected, taking into account the difficulties in the absolute calibration of the thermal emission data, the uncertainties in the local inversion, and the extrapolations that need to be done. Another approach relies on the local polarization phase curve, also retrieved at a constant solar distance of 1.5 AU (see figure 1-b in Levasseur-Regourd, 1996). The overall shape of the curve is smooth, and similar to that of other atmosphereless bodies; the slope at inversion is of the order of 0.20 percent per degree, a value comparable to the values obtained for cometary dust or for $\mathrm{C}$ type asteroids. With the empirical slope at inversion versus albedo relationship (KenKnight et al., 1967), the geometric albedo is found to be in the 0.06 to 0.09 range. The geometric albedo can thus be estimated to be of the order of $(0.13 \pm$ $0.07)$ at $1.5 \mathrm{AU}$, or, taking into account the radial dependence power law, of the order of $(0.15 \pm 0.08)$ at $1 \mathrm{AU}$. The albedo of the dust detected in the asteroidal dust bands is of the order of 0.22 (Spiesman et al., 1995), while the albedo of cometary dust has been found to be in the 0.07 to 0.09 range, by fitting in-situ data with a dynamical model (LevasseurRegourd et al., 1998). The proposed value for the interplanetary dust albedo agrees perfectly with the models that suggest asteroids and comets to be the two main sources, of comparable magnitude, of dust between the orbits of Mars and the Earth (Fechtig, 1989; Dermott, 1994).

\section{Zodiacal Light, What is Still Questionable}

First of all, the question of the stability of the zodiacal light on the scale of more than a few millennia or even centuries is still completely open. Various sources of dust have been identified, such as cometary dust, asteroidal dust and interstellar dust, together with various sinks such as Poynting-Robertson drag, evaporation and collisions; but it is still impossible to demonstrate that the cloud is (or is not) stable over long periods of time. Secondly, the question of the precise location of the tilted symmetry surface is also open. The inclination has been demonstrated, both from insitu and remote observations, to be greater than that of the invariant plane in the inner solar system (Leinert et al., 1980; James et al., 1997); it is thus likely that the plane of symmetry is rather a warped symmetry surface. Thirdly, changes of the order of a few percent in zodiacal brightness are still poorly documented, although small short time enhancements have been noticed in particular directions by various authors. Prominent features have indeed been detected in the thermal emission, which is most sensitive to changes in the spatial density, while the zodiacal light is also sensitive to changes in optical properties.

Besides the above mentioned unsolved problems, the zodiacal light has not been measured accurately in the near ultraviolet, where it is very low, and in the infrared, where it is contaminated by the thermal emission; it is far from having been measured everywhere in the solar system; and finally the local optical properties of the dust are hardly derived outside of the symmetry plane. It is not surprising that so many open questions remain. Not a single observation has ever been performed out of the ecliptic plane. Besides, except for the Clementine photographs (Cooper et $a l .$, 1996), no space observations of the zodiacal light have been performed anymore since the early eighties, although the instrumental techniques have dramatically improved. The models (e.g. Divine, 1993), very useful to compare or to fit the data, are thus not necessarily realistic, and further observations are definitely needed. However, while we are waiting for new observations, performed with higher accuracy, new tools are being developed, which should allow the retrieval of the physical properties of the dust from the data already available.

\section{Towards a Determination of the Physical Properties of the Dust}

A logical method to derive the properties of the dust from the zodiacal light observations is to compare the phase curves derived from observations with those obtained by light scattering computations. The information provided by dust collection and impact indicates that the dust particles are heterogeneous aggregates of submicron sized particles, whose size is greater than a few tens of microns; the Mie 


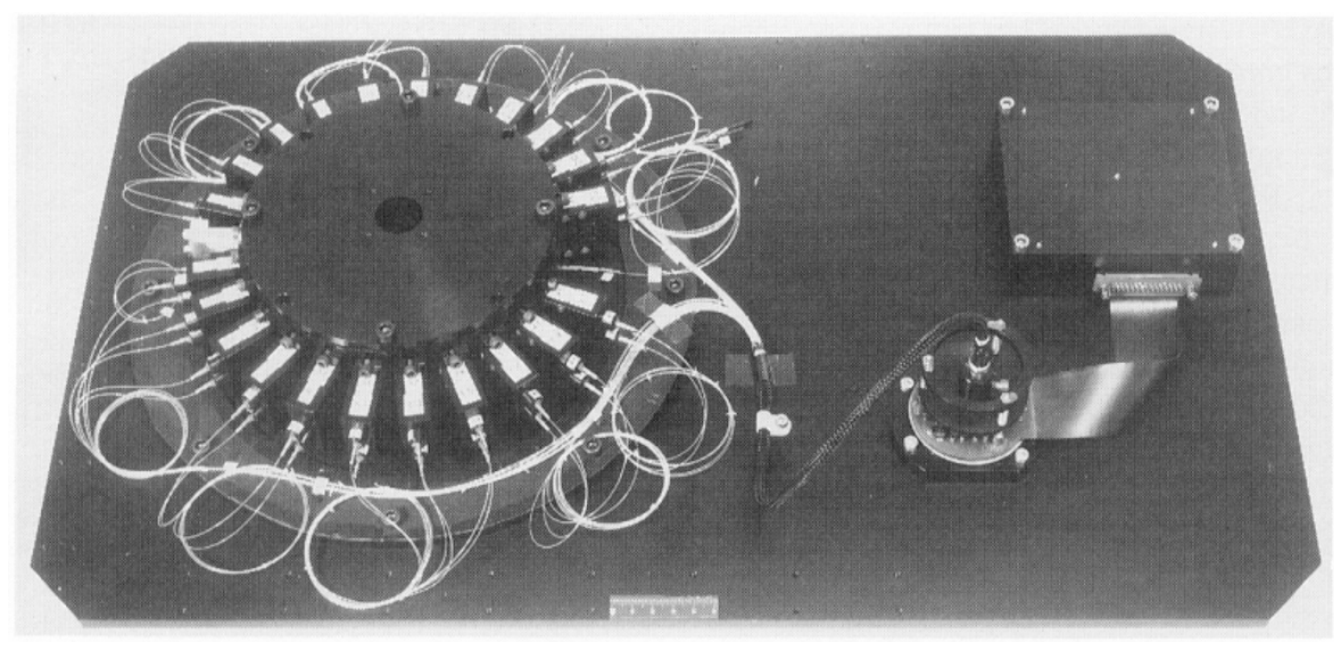

(a)

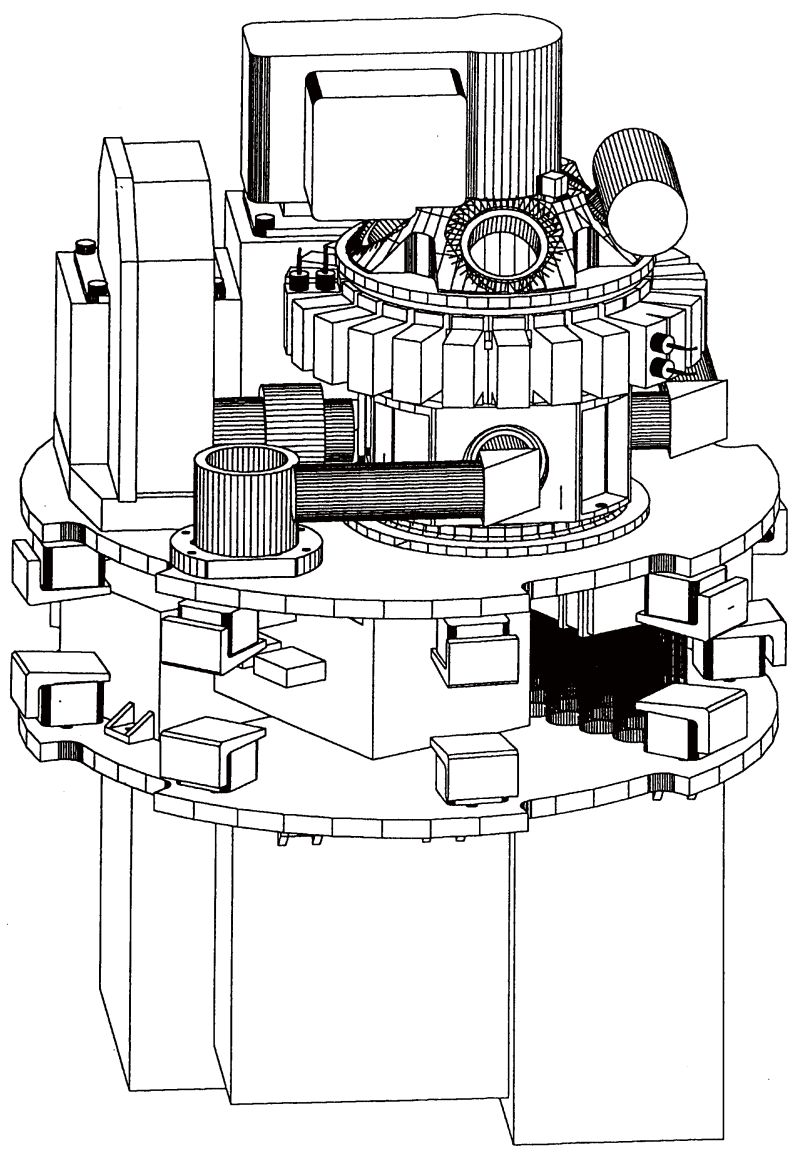

(b)

Fig. 1. The CODAG experiment monitors the aggregation of homogeneously dispersed micrometric dust particles inside a low pressure chamber under microgravity conditions. The CODAG/LSU unit measures simultaneously the brightness and polarization phase curves of the dust particles and aggregates, through 22 analyzer blocks equally distributed along the $180 \mathrm{~mm}$ diameter equatorial ring. The instrument, developed by Fokker Space, will fly on board an ESA rocket; it could be a prototype for an experiment on board Mir and/or the International Space Station to provide some links between the optical and physical properties of the various populations of zodiacal dust. (a) CODAG/ LSU laboratory model: LSU ring and its analyzers on the left, CCD detector which records the polarized components on the right. (b) CODAG and CODAG/LSU implantation: from top to bottom, dust injection device, LSU ring with two microscopes, and electronic boxes. theory can thus hardly be used. Besides some other computational methods, the Draine and Flatau code (1994) has been used successfully by various teams to derive the scattering properties of complex particles through the Discrete Dipole Approximation (e.g. Xing and Hanner, 1996; Levasseur-Regourd et al., 1997). We have noticed, with hundred of monomers and size parameters up to 7, that fluffy particles of fractal dimension 2 reproduce the trends observed for the polarimetric phase curves, once an index similar to that proposed by Mukai et al. (1987), or possibly with a greater imaginary part, is chosen. However, scattering by very large dust particles cannot yet be modeled, and a "ground truth" needs to be obtained to demonstrate the validity of the computational assumptions.

An alternative method is to perform laboratory measurements. Light scattering on dust layers which are sedimented by the Earth's gravity is not representative of the scattering on a low density cloud. Elaborate techniques of levitation have been successfully developed; they unfortunately forbid the use of some particles, for instance elongated particles, whose polarization would be modified after alignment in a jet, and do not reproduce the velocity field which prevails in the interplanetary medium. Most promising simulations have been obtained by microwave analog techniques (Gustafson, 1996); as for numerical models, they suppose that the problem is already partly solved, and assume the shape and the structure of the scattering particle or aggregate.

We have proposed, in a complementary approach, to develop a light scattering facility reproducing the natural medium, under low pressure and microgravity conditions. A three-step development is being made. Firstly, parabolic flights have demonstrated the feasibility of such measurements. The PROGRA² (PRopriétés Optiques des GRains Astronomiques et Atmosphériques) experiment, on board the CNES Caravelle, the NASA KC 135 and now the CNES Airbus, has allowed us to obtain, for various dust samples of a few tens of microns, significant polarimetric phase curves (Worms et al., 1996). Secondly, a rocket flight instrument has been designed to monitor the evolution of the brightness and polarimetric phase curves, as the particles are colliding and possibly aggregating (Fig. 1). The LSU (Light Scattering Unit) will operate on board an ESA Maser rocket, jointly 
with the CODAG (COsmic Dust AGgregation) experiment, which disperses homogeneously the dust sample in a thin gas atmosphere and monitors the aggregation process with a 3D scanning long distance microscopy system (Blum, 1995; Levasseur-Regourd et al., 1997). Thirdly, a facility such as OPAL (Optical Properties of Aggregates in Levitation), dedicated to systematic light scattering measurements for dust particles and aggregates, has been suggested for long duration operations on board Mir and/or the International Space Station (Levasseur-Regourd et al., 1996; Blum et al., 1996). It would, amongst other objectives, provide the missing links between the optical properties (e.g. albedo, polarization) and physical properties (e.g. porosity, size distribution, bulk density) of the various populations of dust which build up the zodiacal cloud, and provide clues to their temporal evolution.

Acknowledgments. The PROGRA ${ }^{2}$ and CODAG/LSU experiments have been developed under CNES and ESA contracts. The OPAL project has been studied under a CNES phase A study.

\section{References}

Blum, J., Laboratory and space experiments to study pre-planetary growth, Adv. Space Res., 15(10), 39-54, 1995.

Blum, J., T. Henning, M. Cabane, M. Fonda, F. Giovane, B. A. S. Gustafson, H. U. Keller, W. Markiewicz, A. C. Levasseur-Regourd, J. C. Worms, J. A. Nuth, and F. Rogers, The concept of a facility for cosmic dust research on the International Space Station, ESA Symposium on Space Station Utilization, ESA SP-385, pp. 303-308, 1996.

Cooper, B. L., H. A. Zook, and A. E. Potter, Clementine photographs of the inner zodiacal light, in Physics, Chemistry, and Dynamics of Interplanetary Dust, edited by B. A. S. Gustafson and M. S. Hanner, pp. 333-336, Astronomical Society of the Pacific, 104, San Francisco, 1996.

Dermott, S. F., S. Jayaraman, Y. L. Xu, B. A. S. Gustafson, and J. C. Liou, Circumsolar ring of asteroidal dust in resonant lock with the Earth, Nature, 369, 719-723, 1994.

Divine, N., Five populations of interplanetary meteoroids, J. Geophys. Res., 98, 17029-17048, 1993.

Draine, B. T. and P. J. Flatau, Discrete dipole approximation for scattering calculations, J. Opt. Soc. Am. A., 11(4), 1491-1499, 1994.

Dumont, R. and A. C. Levasseur-Regourd, Zodiacal light photopolarimetry IV, annual variations of brightness and the symmetry plane of the zodiacal cloud, Astron. Astrophys., 64, 9-16, 1978.

Dumont, R. and A. C. Levasseur-Regourd, Properties of interplanetary dust from infrared and optical observations I: temperature, global volume intensity, albedo and their heliocentric gradients, Astron. Astrophys., 191, 154-160, 1988.

Dumont, R. and F. Sanchez, Zodiacal light photopolarimetry I and II, Astron. Astrophys., 38, 397-412, 1975.

Dumont, R. and F. Sanchez, Zodiacal light photopolarimetry III, Astron. Astrophys., 51, 393-399, 1976.

Fechtig, H., Dust in the solar system, Z. Naturforsch., 44a, 877-882, 1989.

Gustafson, B. A. S., Optical properties of dust from laboratory scattering measurements, in Physics, Chemistry, and Dynamics of Interplanetary Dust, edited by B. A. S. Gustafson and M. S. Hanner, pp. 401-408, Astronomical Society of the Pacific, 104, San Francisco, 1996.

Hanner, M. S., J. G. Sparrow, J. L. Weinberg, and D. E. Beeson, Pioneer 10 observations of zodiacal light brightness near the ecliptic: changes with heliocentric distance, in Interplanetary Dust and Zodiacal Light, edited by H. Elsässer and H. Fechtig, pp. 29-35, Lectures notes in physics 48, Springer-Verlag, Heidelberg, 1976.

James, J. F., T. Mukai, T. Watanabe, M. Ishiguro, and R. Nakamura, The morphology and brightness of the zodiacal light and gegenschein, MNRAS, 288(4), 1022-1026, 1997.

KenKnight, C. E., D. L. Rosenberg, and G. K. Wehner, Parameters of the optical properties of the lunar surface powder in relation to solar-wind bombardment, J. Geophys. Res., 72, 3105-3129, 1967.
Leinert, C. and E. Pitz, Zodiacal light observed by Helios through solar cycle No. 21, Astron. Astrophys., 210, 399-402, 1989.

Leinert, C., M. Hanner, I. Richter, and E. Pitz, The plane of symmetry of interplanetary dust in the inner solar system, Astron. Astrophys., 82, 328336, 1980.

Leinert, C., I. Richter, E. Pitz, and M. Hanner, Helios zodiacal light measurements, a tabulated summary, Astron. Astrophys., 110, 355-357, 1982.

Leinert, C., S. Bowyer, L. Haikala, M. Hanner, M. G. Hauser, A. C. Levasseur-Regourd, I. Mann, K. Mattila, W. T. Reach, W. Schlosser, J. Staude, G. N. Toller, J. L. Weiland, J. L. Weinberg, and A. Witt, The 1997 reference of diffuse nightsky brightness, Astron. Astrophys., Suppl., 127, 1-9, 1998.

Levasseur-Regourd, A. C., Optical and thermal properties of zodiacal dust, in Physics, Chemistry, and Dynamics of Interplanetary Dust, edited by B. A. S. Gustafson and M. S. Hanner, pp. 301-308, Astronomical Society of the Pacific, 104, San Francisco, 1996.

Levasseur-Regourd, A. C. and R. Dumont, Absolute photometry of zodiacal light, Astron. Astrophys., 84, 277-279, 1980.

Levasseur-Regourd, A. C., J. B. Renard, and R. Dumont, The zodiacal cloud complex, in Origin and Evolution of Interplanetary Dust, edited by A. C. Levasseur-Regourd and H. Hasegawa, pp. 131-138, Kluwer, Dordrecht, 1991,

Levasseur-Regourd, A. C., J. Blum, T. Henning, T. Poppe, M. Cabane, V. Haudebourg, P. Rannou, and J. C. Worms, OPAL, a light scattering facility for optical measurements of dust samples, ESA Symposium on Space Station Utilization, ESA SP-385, pp. 401-404, 1996.

Levasseur-Regourd, A. C., M. Cabane, J. C. Worms, and V. Haudebourg, Physical properties of dust in the solar system: relevance of the computational approach and of measurements under microgravity conditions, Adv. Space Res., 20, 8, 1585-1594, 1997.

Levasseur-Regourd, A. C., M. Fulle, N. McBride, and E. Hadamcik, Constraints on the physical properties of cometary dust particles derived from in situ measurements, Astron. Astrophys., 1998 (submitted).

Mann, I., The solar F-corona: calculations of the optical and infrared brightness of circumsolar dust, Astron. Astrophys., 261, 329-335, 1992.

Mann, I., Dust near the Sun, in Physics, Chemistry, and Dynamics of Interplanetary Dust, edited by B. A. S. Gustafson and M. S. Hanner, pp. 315-320, Astronomical Society of the Pacific, 104, San Francisco, 1996.

Mukai, T., S. Mukai, and S. Kikuchi, Complex refractive index of grain material deduced from the visible polarimetry of comet $\mathrm{P} / \mathrm{Halley}$, Astron. Astrophys., 187, 650-652, 1987.

Renard, J. B., A. C. Levasseur-Regourd, and R. Dumont, Properties of interplanetary dust from infrared observations II: brightness, polarization, temperature, albedo and their dependence on the elevation above the ecliptic, Astron. Astrophys., 304, 602-608, 1995.

Renard, J. B., R. Dumont, A. C. Levasseur-Regourd, and E. Hadamcik, Clues in zodiacal light observations for a dust ring along the Earth's orbit, in Physics, Chemistry, and Dynamics of Interplanetary Dust, edited by B. A. S. Gustafson and M. S. Hanner, pp. 329-332, Astronomical Society of the Pacific, 104, San Francisco, 1996.

Spiesman, W. J., M. G. Hauser, T. Kelsall, C. M. Lisse, S. H. Moseley, W. T. Reach, R. F. Silverberg, S. W. Stemwedel, and J. L. Weiland, Near and far infrared observations of interplanetary dust bands from the COBE diffuse infrared background, Astrophys. J., 442, 662-667, 1995.

Toller, G. N. and J. L. Weinberg, The change in near-ecliptic zodiacal light brightness with heliocentric distance, in Properties and Interactions of Interplanetary Dust, edited by R. H. Giese and P. Lamy, pp. 21-25, Reidel, Dordrecht, 1985.

Worms, J. C., A. C. Levasseur-Regourd, E. Hadamcik, and D. Bourras, Scattering properties of dust particles in weightlessness, in Physics, Chemistry, and Dynamics of Interplanetary Dust, edited by B. A. S. Gustafson and M. S. Hanner, pp. 415-418, Astronomical Society of the Pacific, 104, San Francisco, 1996.

Xing, Z. and M. S. Hanner, Modelling the temperature of cometary particles, in Physics, Chemistry, and Dynamics of Interplanetary Dust, edited by B. A. S. Gustafson and M. S. Hanner, pp. 437-441, Astronomical Society of the Pacific, 104, San Francisco, 1996.

A. Ch. Levasseur-Regourd (e-mail: aclr@aerov.jussieu.fr) 